\title{
Editorial for the special issue on metaheuristics for combinatorial optimization
}

\author{
Bin $\mathrm{Hu}^{1}$ - Francisco Chicano ${ }^{2}$
}

Published online: 8 July 2017

(C) Springer Science+Business Media, LLC 2017

Combinatorial optimization is a discipline in computer science and applied mathematics. The goal is to find an optimal solution from a finite, but typically exponentially large set, according to an objective function. If there is more than one objective, we speak of multiobjective combinatorial optimization and the goal is to find a set of Pareto-optimal solutions. Combinatorial optimization problems arise widely in industry and academia and, unfortunately, many of them are NP-hard and no polynomial time algorithm can guarantee their solution to a certified optimality unless $P=N P$. Techniques for solving these problems can roughly be classified into two main categories: exact and heuristic algorithms. Exact algorithms are guaranteed to find an optimal solution and to prove its optimality. However, the run-time often increases dramatically with a problem instance's size, and therefore they are less applicable for real-world applications. For larger instances it is reasonable to use heuristic or metaheuristic algorithms, which are designed to obtain good but not necessarily optimal solutions in acceptable time. These methods are the primary scope of this special issue.

The papers in this issue have been selected among 56 submissions after a thorough peer review process. The final selection contains 12 high quality papers addressing practical applications and theoretical analyses of metaheuristic algorithms in the context of combinatorial optimization problems. Six of them are extended versions of conference papers from the 16th European Conference on Evolutionary Computation in Combinatorial Optimisation (EvoCOP 2016). The contents of this issue, outlined in

\footnotetext{
$凶$ Francisco Chicano chicano@1cc.uma.es

$\mathrm{Bin} \mathrm{Hu}$

bin.hu@ait.ac.at

1 AIT Austrian Institute of Technology, Vienna, Austria

2 University of Malaga, Malaga, Spain
} 
the next paragraphs, reflect the diversity of the application domains and the methods applied to solve the problems.

Local Optima Networks (LONs) have shown to be a useful tool to visualize the landscape structure of combinatorial optimization problems. In a LON, the nodes are local optima of the problem and an edge exists between two nodes if it is possible to reach one local optima from the other using some kind of perturbation operator. Using the LON and the transition probabilities between basins of attraction, it is possible to estimate the probability of finding the global optimum. In the paper "Search Difficulty and PageRank Centrality in Local Optima Networks", Sebastian Herrmann, Gabriela Ochoa and Franz Rothlauf show that PageRank centrality is a good indicator to predict the performance of local search based metaheuristics.

LONs are also used to analyze the landscape structure of the Traveling Salesperson Problem in "Mapping the Global Structure of TSP Fitness Landscapes", by Gabriela Ochoa and Nadarajen Veerapen. In this work the Lin-Kernighan algorithm is used to define a neighborhood and the double-bridge operator is used as perturbation. New ways of visualizing LONs are explored in this paper, revealing that multiple funnels exist in TSP and contradicting the big-valley hypothesis.

The Traveling Thief Problem (TTP) is a problem combining the Knapsack Problem an the Traveling Salesperson Problem. It was proposed to model the case, very common in real world, in which a combinatorial optimization problem is a combination of two well-known underlying problems. In "A case study of algorithm selection for the traveling thief problem", by Markus Wagner, Marius Lindauer, Mustafa Misir, Samadhi Nallaperuma and Frank Hutter, a set of features for the TTP instances are defined and used as the base of an algorithm selection framework. The algorithm portfolio of the framework contains 21 algorithms. An experimental evaluation with 9720 instances of TTP reports substantial improvements over single algorithms and highlights the algorithms contributing more to the algorithm portfolio.

Graph coloring problems are at the core of many real-world applications, like frequency assignment, register allocation or timetabling. A dynamic version of graph coloring, where the vertices are fixed but the edges change over time, is the focus of "Tackling the edge dynamic graph colouring problem with and without future adjacency information" by Bradly Hardy, Rhyd Lewis and Jonathan Thompson. The paper combines Tabu Search with new methods to transform a feasible coloring at time $t$ to a coloring for time $t+1$. This transformation is done in two scenarios: when there is no information about the change and when the probability of an edge to be present in the future graph is known.

The bin packing problem consists in assigning a set of items to bins such that the number of bins used is minimized and the items added to any bin do not exceed the capacity of the bin. In the case of the 2-Constraint bin packing problem, there are two "capacities" associated to each bin and two "weights" associated to each item. Roberto Aringhieri, Davide Duma, Andrea Grosso, and Pierre Hosteins propose two new algorithms for solving this problem in "Simple but effective heuristics for the 2Constraint Bin Packing Problem". The first one is a variant of the Best First Decreasing heuristic and the second one is a multi-start neighborhood search.

Network design problems, despite their theoretic appearance, lay the foundation of many real-world applications. A good example is the paper "A Constraint-based 
Parallel Local Search for Edge Disjoint Rooted Distance-Constrained Minimum Spanning Tree Problem" by Alejandro Arbelaez, Deepak Mehta, Barry O'Sullivan and Luis Quesada. The abstract problem can be used to model VLSI circuit design, QoS routing or traffic engineering where fault tolerance is considered. The authors use a constraint-based parallel local search algorithm to tackle problem instances derived from real-world optical communication networks in Ireland, Italy and the UK.

Multimedia communications require a large bandwidth in computer networks that is mitigated by the use of multicast routing. In this context, the multimedia streams with origin in some nodes of the network are distributed along a set of trees defined over the network. These trees change with time when nodes join/leave a multicast group or communication links fail. In "Dynamic Reconfiguration in Multigroup Multicast Routing under Uncertainty", Pavel Troubil, Hana Rudová and Petr Holub, propose the use of Ant Colony Optimization to compute the trees in multicast routing in such a way that the number of source-destination paths affected by a change is minimized, thus reducing the number of disruptions in the multimedia communications.

Web service composition is also a relatively recent field of research and deals with paradigms for building distributed computing applications over the internet. In the paper "Evolutionary Computation for Automatic Web Service Composition-An Indirect Representation Approach", Alexandre Sawczuk da Silva, Yi Mei, Hui Ma and Mengjie Zhang propose an evolutionary approach with an indirect encoding. The genotype, a sequence of services, is mapped into one of several generic composition schemes. By evolving them with evolutionary techniques, the quality is assigned to the original sequence.

Integer exponentiation is one of the basic operations in cryptographic algorithms. It should be efficiently implemented, since it should run in small hardware devices. Given an exponent, the problem of finding the optimal sequence of multiplications that results in the exponent is NP-hard. In "Finding Short and Implementation-friendly Addition Chains with Evolutionary Algorithms", Stjepan Picek, Carlos A. Coello Coello, Domagoj Jakobovic, and Nele Mentens propose the use of a Genetic Algorithm with a novel encoding to find the optimal sequence of multiplications.

A nowadays relevant topic in counter terrorism is the strategic placement of suicide bomber detectors. Since these detectors are not fully reliable, the goal is to place them in a given area so that the expected number of casualties in case of an attack is minimized. In the paper "Metaheuristic Approaches to the Placement of Suicide Bomber Detectors" by Carlos Cotta and José Enrique Gallardo, different metaheuristic approaches are presented and evaluated against a greedy heuristic from the literature. The authors also conduct a detailed sensitivity analysis and test the approach on instances built after realistic scenarios such as curvy streets and small squares.

The vehicle routing problem has numerous applications in the real world, both for passenger transportation and freight transportation. A rather novel aspect is to consider alternative routes, which is addressed in the paper "On strategies for a candidate set of routing alternatives in the bi-objective $k$-dissimilar vehicle routing problem" by Sandra Zajac. The goal is to find a set of $k$ dissimilar alternatives where the tradeoff between minimizing the longest routing and maximizing the minimum dissimilarity between two routes is investigated. Since these are conflicting objectives, a two-phase heuristic is proposed which computes a Pareto-set. 
The repetition-free longest common subsequence problem is a classic NP-hard problem in bio-informatics. In "A Comprehensive Comparison of Metaheuristics for the Repetition-Free Longest Common Subsequence Problem" by Christian Blum and Maria J. Blesa, existing approaches in the literature are analyzed and compared in detail. The authors combine the two best algorithms-Construct, Merge, Solve \& Adapt heuristic and a Beam-ACO_into a hybrid approach and show that it benefits greatly from the synergy effects. For comprehensive testing, the standard benchmark set is extended by larger instances in order to make them more challenging.

Finally, we would like to thank all the authors submitting a paper to this special issue (accepted or not), the referees for their constructive and thorough reviews, and the editor-in-chief, Manuel Laguna, for his great and prompt support. 